\title{
Incremental prognostic value of left ventricular function analysis over non-invasive coronary angiography with multidetector computed tomography
}

Fleur R. de Graaf, MD, ${ }^{a}$ Jacob M. van Werkhoven, MSc, ${ }^{\mathrm{a}, \mathrm{b}}$ Joëlla E. van Velzen, $M D,{ }^{\mathrm{a}, \mathrm{b}} M$. Louisa Antoni, MD, ${ }^{\mathrm{a}}$ Mark J. Boogers, $M D,{ }^{\mathrm{a}, \mathrm{b}}$ Lucia J. Kroft, $\mathrm{MD}, \mathrm{PhD},{ }^{\mathrm{c}}$ Albert de Roos, MD, PhD, ${ }^{c}$ Martin J. Schalij, MD, PhD, ${ }^{a} J$. Wouter Jukema, MD, PhD, ${ }^{a, b}$ Ernst E. van der Wall, MD, PhD, ${ }^{a, b}$ Joanne D. Schuijf, PhD, ${ }^{a}$ and Jeroen J. Bax, MD, PhD ${ }^{\mathrm{a}}$

Background. The purpose of this study was to determine the prognostic value of computed tomography coronary angiography (CTA)-derived left ventricular (LV) function analysis and to assess its incremental prognostic value over the detection of significant stenosis using CTA.

Methods. In 728 patients (400 males, mean age $55 \pm 12$ years) with known or suspected CAD, the presence of significant stenosis ( $\geq 50 \%$ stenosis) and $L V$ function were assessed using CTA. LV end-systolic volume (LVESV), LV end-diastolic volume (LVEDV), and LV ejection fraction (LVEF) were calculated. LV function was assessed as a continuous variable and using cutoff values (LVEDV > $215 \mathrm{~mL}$, LVESV > $90 \mathrm{~mL}, \mathrm{LVEF}<49 \%$ ). The following events were combined in a composite end-point: all-cause mortality, non-fatal myocardial infarction, and unstable angina pectoris requiring hospitalization.

Results. On CTA, a significant stenosis was observed in 221 patients (30\%). During followup [median 765 days, 25-75th percentile: 493-978] an event occurred in 45 patients $(6.2 \%)$. After multivariate correction for clinical risk factors and CTA, LVEF $<49 \%$ and LVESV > $90 \mathrm{~mL}$ were independent predictors of events with an incremental prognostic value over clinical risk factors and CTA.

Conclusions. The present results suggest that LV function analysis provides independent and incremental prognostic information beyond anatomic assessment of CAD using CTA. (J Nucl Cardiol 2010;17:1034-40.)

Key Words: Left ventricular function $\cdot$ computed tomography $(\mathrm{CT}) \cdot$ prognosis

\section{INTRODUCTION}

Multidetector computed tomography coronary angiography (CTA) has emerged as an important non-

From the Department of Cardiology, ${ }^{\text {a }}$ Leiden University Medical Center, Leiden, The Netherlands; The Interuniversity Cardiology Institute of the Netherlands, ${ }^{\mathrm{b}}$ Utrecht, The Netherlands; Department of Radiology, ${ }^{\mathrm{c}}$ Leiden University Medical Center, Leiden, The Netherlands.

Received for publication Mar 2, 2010; final revision accepted Jul 18, 2010.

Reprint requests: Jeroen J. Bax, MD, PhD, Department of Cardiology, Leiden University Medical Center, Albinusdreef 2, 2333 ZA Leiden, The Netherlands; j.j.bax@lumc.nl.

$1071-3581 / \$ 34.00$

Copyright (c) 2010 The Author(s). This article is published with open access at Springerlink.com

doi:10.1007/s12350-010-9277-4 invasive imaging modality by providing direct anatomic assessment of coronary artery disease (CAD). ${ }^{1-3}$ Recently, several studies have shown that, in addition to its value for the diagnosis of CAD, stenosis detection with CTA may also be useful for risk stratification. ${ }^{4-7}$ Furthermore, besides the assessment of coronary anatomy, left ventricular (LV) function may be evaluated using information derived from the same CTA dataset. ${ }^{8-10} \mathrm{LV}$ function is an established prognostic marker, as has been demonstrated using several imaging modalities including left ventriculography, echocardiography, magnetic resonance imaging (MRI), and single-photon emission computed tomography (SPECT). ${ }^{11-14}$ However, no data are currently available concerning the prognostic significance of CTA-derived LV function assessment. The purpose of this study was therefore to determine the 
prognostic value of CTA-derived LV function analysis and to assess its incremental prognostic value over the detection of significant coronary artery stenosis using CTA.

\section{METHODS}

The study group consisted of consecutive patients with suspected or known CAD who were clinically referred for CTA because of chest pain, a positive or inconclusive exercise electrocardiogram (ECG) test, or an elevated risk profile for cardiovascular disease. The study population is part of a large ongoing registry exploring the prognostic value of CTA. ${ }^{15}$ For the current analysis, only patients with a CTA examination of diagnostic image quality and with additional LV function data were included. Exclusion criteria for CTA examination were (1) (supra)ventricular arrhythmias, (2) renal failure (glomerular filtration rate $<30 \mathrm{~mL} /$ minutes), (3) known allergy to iodine contrast material, (4) severe claustrophobia, or (5) pregnancy.

\section{CTA Data Acquisition}

CTA studies were performed using a 64-row $(\mathrm{N}=647)$ or 320-row $(\mathrm{N}=81)$ multidetector scanner (Aquilion 64 , and Aquilion ONE, respectively, Toshiba Medical Systems, Otawara, Japan). One hour prior to the examination, a single dose of oral beta-blocker medication was administered to patients with a heart rate $\geq 65 \mathrm{bpm}$, unless contraindicated. Based on a pre-defined beta-blocking medication administration protocol, patients with a heart rate between 65 and $75 \mathrm{bpm}$ received $50 \mathrm{mg}$ metoprolol, whereas patients with a heart rate $\geq 75 \mathrm{bpm}$ received $100 \mathrm{mg}$ metoprolol. The total amount of non-ionic contrast media (Iomeron 400; Bracco, Milan, Italy) injected into the antecubital vein was $60-100 \mathrm{~mL}$ (depending on body weight and scanner type) at a flow rate of 5.0 or $6.0 \mathrm{~mL} / \mathrm{second}$, followed by a saline flush of $25-50 \mathrm{~mL}$. In order to synchronize the arrival of the contrast media, bolus arrival was detected using a real-time bolus tracking technique. ${ }^{16}$ All images were acquired during a single inspiratory breath-hold of maximally 12 seconds. For 64-row CTA, a helical-scanning technique was used as previously described. ${ }^{17,18}$ In brief, during the scan, the ECG was registered simultaneously for retrospective gating of the data. A collimation of $64 \times .5 \mathrm{~mm}$ was used. Additional scan parameters were 400 or 500 ms gantry rotation time depending on cardiac frequency, $120 \mathrm{kV}$ tube voltage, and 300-350 mA (depending on body mass index, (BMI) and thoracic geometry). During 320-row CTA, the ECG was registered simultaneously for prospective triggering of the data. In order to perform LV function analysis, ECG-triggered tube modulation was used, as previously described. ${ }^{19}$

The entire heart was imaged in a single heart beat, attaining maximal tube current during $75 \%$ of R-R interval (in patients with stable heart rate $<60 \mathrm{bpm}$ ), during $65-85 \%$ of $\mathrm{R}-\mathrm{R}$ interval (in patients with a heart rate $60-65 \mathrm{bpm}$ ), or during $30-80 \%$ of $\mathrm{R}-\mathrm{R}$ interval (in patients with a heart rate $>65 \mathrm{bpm}$ ). Outside the pre-defined interval, tube current was $25 \%$ of the maximal tube current. In patients with a heart rate $>65 \mathrm{bpm}$ images were acquired during multiple heart beats (typically two). A collimation of $320 \times .5 \mathrm{~mm}$ was used. Additional scan parameters were $350 \mathrm{~ms}$ gantry rotation time, $120-135 \mathrm{kV}$ tube voltage, and 400-580 mA (depending on BMI and thoracic geometry). For the 64-row CTA scanner, the estimated average radiation dose for CTA was $17.6 \pm$ $5.6 \mathrm{mSv}$. For the 320-row CTA scanner, the estimated average radiation dose for single heart beat CTA's using ECG-triggered tube modulation was $10.7 \pm 3.6 \mathrm{mSv}$. In patients, in whom CTA image acquisition was performed during multiple heart beats, average estimated radiation dose was $16.7 \pm 6.3 \mathrm{mSv}$.

Coronary arteries were evaluated using the reconstruction dataset with the least motion artifacts, typically acquired during an end-diastolic phase. To assess LV function and LV volumes, ten series of $2.0-\mathrm{mm}$ slices were reconstructed from the same dataset at every $10 \%$ throughout the cardiac cycle, starting at early systole ( $0 \%$ of cardiac cycle) to end-diastole (90\% of cardiac cycle).

\section{Data Analysis}

CTA and LV function reconstructions were transferred to a remote workstation with dedicated analysis software (for 64-row CTA reconstructions: Vitrea 2, Vital Images, Minnetonka, MN, USA; for 320-row CTA reconstructions: Vitrea FX 1.0, Vital Images). The presence of coronary stenosis was assessed by scrolling through axial images, combined with visual assessment of curved multiplanar reconstructions in $\geq 2$ orthogonal planes. CTA examinations were evaluated on a patient basis for the presence of a significant stenosis $(\geq 50 \%$ luminal narrowing) by two experienced observers. Discrepancies in interpretation were resolved by consensus.

For the purpose of $\mathrm{LV}$ function analysis, appropriate phases for LV end-systolic volume (LVESV) and LV enddiastolic volume (LVEDV) were acquired, by selecting the smallest and largest cross-sectional LV cavity areas, respectively. Upper limit of the LV was determined at the basal level of the mitral valve and the start of the LV outflow tract, as previously described. ${ }^{20}$ Endocardial borders were outlined using a semi-automated method from the base to the apex on the short-axis cine images by an independent observer. Papillary muscles were excluded from the ventricular cavity. The LVEDV and LVESV volumes were calculated and the LVEF was derived by subtracting the LVESV from the LVEDV and dividing the result by the LVEDV. ${ }^{9}$ Observers for LV function analysis were blinded to CTA data.

\section{End-Points}

Patient follow-up data were gathered by a single observer, blinded to the baseline CTA results, using clinical visits and/or standardized telephone interviews. A composite end-point was constructed using the following events: all-cause mortality, non-fatal myocardial infarction, and unstable angina pectoris requiring hospitalization. Non-fatal infarction was defined based on criteria of typical chest pain, elevated cardiac enzyme 
levels, and typical changes on the ECG. ${ }^{21}$ Unstable angina was defined according to the European Society of Cardiology guidelines as acute chest pain with or without the presence of ECG abnormalities, and negative cardiac enzyme levels. ${ }^{22}$

\section{Statistical Analysis}

Statistical analysis was performed using SPSS software (version 16.0, SPSS Inc., Chicago, IL, USA). Quantitative data were expressed as mean $\pm \mathrm{SD}$. Categorical variables were described as numbers and percentages. Cox regression analysis was used to determine the prognostic value of the presence of significant stenosis and LV function parameters on CTA. First, univariate analysis of baseline characteristics, the presence of significant stenosis, and LV function parameters was performed using a composite end-point of all-cause mortality, non-fatal infarction, and unstable angina. For each variable, a hazard ratio (HR) with a 95\% confidence interval (CI) was calculated. Increased LVEDV and LVESV were defined as LVEDV > $215 \mathrm{~mL}, \mathrm{LVESV}>90 \mathrm{~mL}$ and reduced LVEF was defined as LVEF $<49 \%$, as described previously. ${ }^{23}$ These values were determined using the $95 \% \mathrm{CI}$ boundaries for $3 \mathrm{D}$ LV dimensions and function as determined by CTA. ${ }^{23}$ After univariate analysis, several multivariate models were created to correct LV function parameters for clinical risk factors and the presence of significant stenosis on CTA. In case variables showed strong interrelation (Pearson's correlation coefficient $>.8$ ), variables were excluded from the same multivariate model. Finally, the incremental value of LV function variables over clinical risk factors (age, gender, smoking, and known CAD) and the presence of significant stenosis on CTA were assessed by calculating the global $\chi^{2}$ values. A $P$-value $<.05$ was considered statistically significant.

\section{RESULTS}

From the registry, additional LV function measurements (derived from CTA) were available in 813 patients. In 29 patients (3.6\%) the CTA examination was of non-diagnostic image quality and these patients were excluded from the study. Furthermore, a total of 56 patients $(6.9 \%)$ were lost to follow-up. As a result, the patient group consisted of 728 individuals. The main clinical characteristics of the population are listed in Table 1 . In summary, 55\% were male and the mean age was $55 \pm 12$ years.

\section{Baseline CTA and LV Function}

In the study population of 728 patients, a significant stenosis (luminal narrowing $\geq 50 \%$ ) was identified on CTA in 221 patients (30\%). When evaluating LV volumes, an average LVEDV of $138 \pm 38 \mathrm{~mL}$ was observed. For LVESV an average value of $52 \pm 29 \mathrm{~mL}$ was observed. As a result, average LVEF was $64 \pm 10 \%$. Increased LVEDV (> $215 \mathrm{~mL})$ and LVESV $(>90 \mathrm{~mL})$
Table 1. Patient characteristics

\begin{tabular}{ll}
\hline & $\begin{array}{c}\text { Total: } \\
\text { N }=\mathbf{7 2 8}\end{array}$ \\
\hline Gender (male/female) & $400 / 328$ \\
Age (years) & $55 \pm 12$ \\
Reason for referral & \\
Typical chest pain & $83(12 \%)$ \\
Atypical chest pain & $189(26 \%)$ \\
Non-anginal chest pain & $132(18 \%)$ \\
Elevated risk profile & $213(29 \%)$ \\
Positive or inconclusive exercise ECG & $111(15 \%)$ \\
Clinical risk factors & \\
Diabetes & $222(31 \%)$ \\
Hypercholesterolemia* & $261(36 \%)$ \\
Hypertension & $\dagger$ \\
Family history of coronary artery & $317(44 \%)$ \\
$\quad$ disease & $309(42 \%)$ \\
Smoking & \\
Obesity (BMI $\geq 30$ kg/m ${ }^{*}$ ) & $137(19 \%)$ \\
Known CAD & $143(20 \%)$ \\
\hline Sermo & $96(13 \%)$ \\
\hline
\end{tabular}

*Serum total cholesterol $\geq 230 \mathrm{mg} / \mathrm{dL}$ and/or serum triglycerides $\geq 200 \mathrm{mg} / \mathrm{dL}$ or treatment with lipid lowering drugs.

'Defined as systolic blood pressure $\geq 140 \mathrm{mmHg}$ and/or diastolic blood pressure $\geq 90 \mathrm{mmHg}$ and/or the use of antihypertensive medication.

Defined as presence of coronary artery disease in first degree family members at $<55$ years in men and $<65$ years in women.

$B M I$, Body mass index; $C A D$, coronary artery disease.

were present in 31 patients $(4.3 \%)$ and 50 patients (6.9\%), respectively. A reduced LVEF $(<49 \%)$ was present in 43 patients $(7.3 \%)$.

\section{Follow-up}

The median follow-up period was 765 days (25-75th percentile: 493-978); an event occurred in 45 patients $(6.2 \%)$. All-cause death was reported in 23 patients $(3.2 \%)$, non-fatal myocardial infarction occurred in 7 patients $(1.0 \%)$, and 15 patients $(2.0 \%)$ were admitted to the hospital due to unstable angina.

\section{Survival Analysis}

Univariate analysis of clinical risk factors, significant stenosis on CTA, and LV function analysis parameters is depicted in Table 2. Of the clinical risk factors, age, smoking, and known CAD were significant predictors of events. Also, the presence of a significant 
Table 2. Univariate predictors of events

\begin{tabular}{|c|c|c|}
\hline & $\begin{array}{c}\text { HR } \\
(95 \% \mathrm{CI})\end{array}$ & P-value \\
\hline \multicolumn{3}{|c|}{ Baseline clinical characteristic } \\
\hline Age & $1.04(1.01-1.07)$ & .005 \\
\hline Male gender & $1.36(.75-2.49)$ & .315 \\
\hline Diabetes & $1.03(.55-1.93)$ & .939 \\
\hline Hypercholesterolemia* & $.73(.38-1.39)$ & .333 \\
\hline Hypertension $^{\dagger}$ & $.89(.53-1.73)$ & .958 \\
\hline $\begin{array}{l}\text { Family history of } \\
\text { coronary artery } \\
\text { disease }\end{array}$ & $.61(.32-1.15)$ & .125 \\
\hline Smoking & $2.31(1.25-4.25)$ & .007 \\
\hline $\begin{array}{l}\text { Obesity } \\
\qquad\left(B M I \geq 30 \mathrm{~kg} / \mathrm{m}^{2}\right)\end{array}$ & $.55(.22-1.39)$ & .205 \\
\hline Known CAD & $2.74(1.46-5.13)$ & .002 \\
\hline \multicolumn{3}{|l|}{ CTA } \\
\hline Significant stenosis & $3.52(1.59-7.83)$ & .002 \\
\hline \multicolumn{3}{|c|}{ LV function (continuous) } \\
\hline $\begin{array}{l}\text { LVEDV (per } \\
10 \mathrm{~mL} \text { increase) }\end{array}$ & $1.07(1.00-1.14)$ & .048 \\
\hline $\begin{array}{l}\text { LVESV (per } \\
10 \mathrm{~mL} \text { increase) }\end{array}$ & $1.10(1.03-1.18)$ & .006 \\
\hline LVEF (per \% increase) & $.97(.94-.99)$ & .006 \\
\hline \multicolumn{3}{|l|}{ LV function (categorical) } \\
\hline LVEDV $>215 \mathrm{~mL}$ & $2.89(1.22-6.86)$ & .016 \\
\hline LVESV > $90 \mathrm{~mL}$ & $3.95(1.99-7.84)$ & $<.001$ \\
\hline LVEF $<49 \%$ & $3.82(1.93-7.57)$ & $<.001$ \\
\hline
\end{tabular}

* Serum total cholesterol $\geq 230 \mathrm{mg} / \mathrm{dL}$ and/or serum triglycerides $\geq 200 \mathrm{mg} / \mathrm{dL}$ or treatment with lipid lowering drugs.

${ }^{\dagger}$ Defined as systolic blood pressure $\geq 140 \mathrm{mmHg}$ and/or diastolic blood pressure $\geq 90 \mathrm{mmHg}$ and/or the use of antihypertensive medication.

Defined as presence of coronary artery disease in first degree family members at $<55$ years in men and $<65$ years in women.

$B M I$, Body mass index; $C A D$, coronary artery disease; $C I$, confidence interval; CTA, computed tomography coronary angiography; $H R$, hazard ratio; $L V E D V$, left ventricular enddiastolic volume; $L V E F$, left ventricular ejection fraction; $L V E S V$, left ventricular end-systolic volume.

stenosis on CTA was identified as a significant predictor of events. Regarding LV function, all three parameters (LVEDV, LVESV, and LVEF) were significant univariate predictors when assessed as continuous variables and categorical variables, using predefined cutoff values.

Multivariate models were created correcting for clinical risk factors (age, gender, smoking, and known CAD) and significant stenosis on CTA. The prognostic values of LV volumes and LVEF are shown in Table 3. A total of six models were created. In the first three models, LVEDV, LVESV and LVEF were assessed as
Table 3. Multivariate models for the prediction of events

\section{HR $(95 \% \mathrm{CI}) \quad P$-value}

$\begin{array}{lll}\begin{array}{l}\text { LV function (continuous) } \\ \text { LVEDV* (per } 10 \mathrm{ml}\end{array} & 1.05(.99-1.13) & .123 \\ \quad \text { increase) } & & \\ \quad \begin{array}{lll}\text { LVESV* (per } 10 \mathrm{ml} \\ \quad \text { increase) }\end{array} & 1.06(.98-1.15) & .119 \\ \quad \text { LVEF (per \% increase) } & .98(.96-1.01) & .177 \\ \text { LV function (categorical) } & & \\ \text { LVEDV }>215 \mathrm{~mL}^{*} & 1.98(.79-4.95) & .143 \\ \text { LVESV }>90 \mathrm{~mL}^{*} & 3.11(1.45-6.67) & .004 \\ \text { LVEF }<49 \% * & 2.61(1.22-5.60) & .014\end{array}$

*Corrected for baseline clinical variables (age, gender, smoking, known CAD) and significant stenosis on CTA. $C A D$, Coronary artery disease; $C I$, confidence interval; $H R$, hazard ratio; $L V E D V$, left ventricular end-diastolic volume; $L V E F$, left ventricular ejection fraction; $L V E S V$, left ventricular end-systolic volume.

continuous variables, whereas in the last three models the predictive value of LV function was determined using the pre-defined cutoff values to indicate reduced LV function. Although none of the continuous LV function variables remained independent predictors of events, using the pre-defined cutoff values as markers for reduced LV function, increased LVESV and reduced LVEF provided additional prognostic information over clinical risk factors and significant stenosis on CTA. Figure 1 illustrates the incremental prognostic value, depicted by $\chi^{2}$ value, of LVESV $>90 \mathrm{~mL}$ over age, gender, smoking, known CAD, and significant stenosis on CTA $(P<.05)$. Similarly, Figure 2 shows that LVEF $<49 \%$ has incremental value $(P<.05)$, and thus enhanced risk stratification beyond the detection of significant stenosis using CTA.

\section{DISCUSSION}

The main finding of this study is that CTA-derived LV function may provide important prognostic information beyond the detection of significant stenosis on CTA.

LV volumes and LVEF are used extensively as clinical markers of cardiac function. In patients with known CAD, LV function analysis provides important information for risk assessment and further management. ${ }^{11,24}$ Post-myocardial infarction patients with a normal LVEF have a relatively good prognosis, whereas the risk of future events has been shown to increase with deteriorating $\mathrm{LVEF}^{25} \mathrm{LV}$ function analysis may also be useful in patients with suspected CAD. Using 2D 


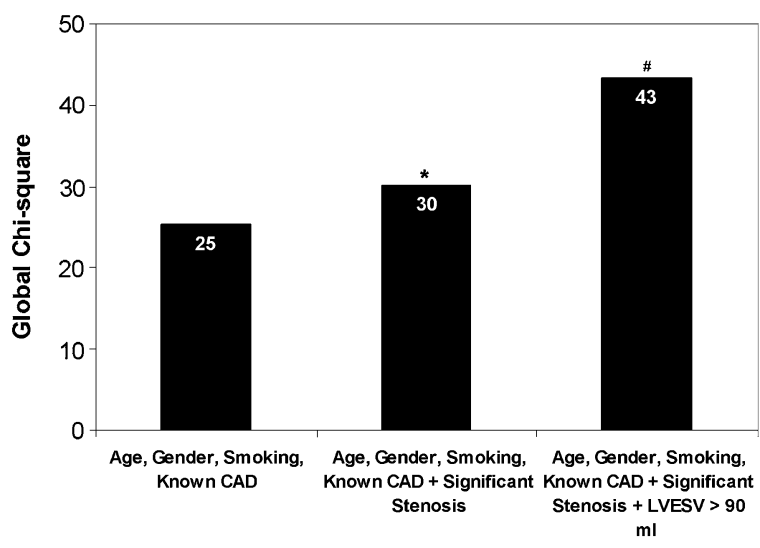

Figure 1. Bar graph illustrating the incremental prognostic value (depicted by $\chi^{2}$ values on the $y$-axis) of LVESV $>90 \mathrm{~mL}$ over age, gender, smoking, known $\mathrm{CAD}$, and significant stenosis. The presence of significant stenosis on CTA has a significant incremental prognostic value over age, gender, smoking, and known CAD (asterisk). A further incremental prognostic value over clinical risk factors and significant stenosis on CTA is observed with the addition of LVESV > $90 \mathrm{~mL}$ (\#). CAD, Coronary artery disease; LVESV, left ventricular end-systolic volume.

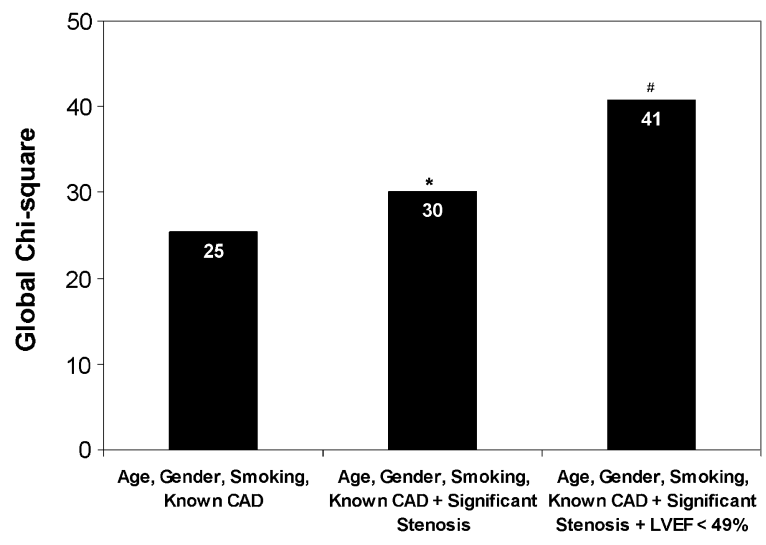

Figure 2. Bar graph illustrating the incremental prognostic value (depicted by $\chi^{2}$ values on the $y$-axis) of LVEF $<49 \%$ over age, gender, smoking, known CAD, and significant stenosis on CTA. The presence of significant stenosis on CTA has a significant incremental prognostic value over age, gender, smoking, and known CAD (asterisk). A further incremental prognostic value over clinical risk factors and significant stenosis on CTA is observed with the addition of LVEF $<49 \%$ (\#). CAD, Coronary artery disease; $L V E F$, left ventricular ejection fraction.

echocardiography in 2,948 patients without prevalent CAD, Devereux et al ${ }^{12}$ showed that LVEF $<40 \%$ was a strong predictor of cardiovascular death (RR 6.9, CI 3.0-15.9, $P<.001$ ) and all-cause mortality (RR 4.8, CI 2.8-8.1, $P<.001$ ), independent of clinical risk factors. In a study by Sharir et al, ${ }^{14}$ evaluating 1,680 patients referred for gated SPECT, LVEF $<45 \%$ and
ESV $>70 \mathrm{~mL}$ were identified as optimal thresholds to accurately stratify patients into high-risk and low-risk groups. Importantly, patients with a normal LVEF or LVESV had a substantially lower annual mortality rate (regardless of the degree of perfusion abnormalities on SPECT) than patients with reduced LVEF or increased LVESV. In this study, the prognostic value of LV function measurements on CTA was evaluated. LV volumes and LVEF provided prognostic information independent of baseline clinical variables. These findings illustrate that measurements of LV function (and volumes) from CTA acquisitions can be beneficial in defining patient risk and if available should be incorporated into the clinical report.

Importantly, patients undergoing CTA are primarily referred for assessment of CAD. As this anatomic information has been established to provide prognostic information, ${ }^{4-6}$ an important objective of this study was to determine the additional value of LV function analysis beyond the detection of significant CAD. Limited information is available regarding the additional prognostic value of $\mathrm{LV}$ function over the non-invasive angiographic assessment of CAD using CTA. In a recent study by Min et $a{ }^{26}{ }^{26}$ evaluating 5,330 consecutive patients without known CAD with a mean follow-up of $2.3 \pm .6$ years, the addition of LV function measured by CTA significantly increased risk correlation for death. Annualized mortality rates in patients with significant CAD and LVEF $\leq 50 \%$ were significantly higher $(3.79 \%)$ than in patients with significant CAD and LVEF $>50 \%$ (1.76\%). Furthermore, Chow et al ${ }^{27}$ recently determined the incremental prognostic value of LV function measured with CTA in 2,076 consecutive patients with a mean follow-up of $16 \pm 8$ months. It was shown that LVEF had incremental prognostic value over CAD severity (HR 1.47, 95\% CI 1.17-1.86). These data indicate that LV function is an important predictor of survival with incremental value to CTA. In line with these results, this study suggests that, although the assessment of significant CAD on CTA is a powerful predictor, LV function parameters provide independent incremental value in predicting adverse events. Thus, the addition of LV function analysis to CTA may further improve risk stratification of patients with known of suspected CAD referred for stenosis detection.

\section{Limitations}

Several limitations of this study merit consideration. First, $6.9 \%$ of patients were lost to follow-up. Second, regional wall motion abnormalities were not assessed in this study. Third, this study is limited by a relatively small patient number. Fourth, CTA is inherently associated with radiation exposure. ${ }^{28}$ As a result, prospective ECG triggering was recently introduced for the purpose of radiation reduction. ${ }^{29,30}$ This technique allows image 
acquisition during a small portion of the cardiac cycle which significantly reduces the radiation exposure. However, as a result data are no longer acquired throughout the entire cardiac cycle, thereby eliminating the possibility of simultaneous LV function analysis from the same dataset. Although LV function may still be assessed, it has become at the expense of increased radiation exposure, also when using ECG-triggered tube current modulation. Therefore, the necessity of LV function analysis using CTA should be carefully weighed against the increase in radiation burden. Importantly, LV function analysis may also be performed using radiation-free modalities, such as echocardiography or MRI. Accordingly, although in this study LV function was derived from CTA, LV function analysis may enhance risk stratification beyond the presence of significant stenosis on CTA regardless of the modality used to derive this information. Additional studies are warranted to gain better understanding of the integration of angiographic and LV function data to refine risk stratification.

\section{Acknowledgments}

Dr. de Graaf was co-supported by the Dutch Technology Foundation STW (Utrecht, the Netherlands), applied science division of NWO and the Technology Program of the Ministry of Economic Affairs, Grant No. 10084. Mr. van Werkhoven was financially supported by a research grant from the Netherlands Society of Cardiology (Utrecht, The Netherlands). Dr. van Velzen was supported by the Netherlands Heart Foundation (The Hague, The Netherlands), Grant No. 2007B223. Dr. Boogers was supported by the Dutch Heart Foundation, Grant 2006T102. Dr. Schalij has research grants from Boston Scientific (Natick, USA), Medtronic (Minneapolis, USA) and Biotronik (Berlin, Germany). Dr. Bax has research grants from Medtronic (Minneapolis, USA), Boston Scientific (Natick, USA), BMS medical imaging (New York, USA), St. Jude Medical (St. Paul, USA), GE Healthcare (Chalfont St. Giles, UK), Biotronik (Berlin, Germany), and Edwards Lifesciences (Irvine, USA).

\section{Open Access}

This article is distributed under the terms of the Creative Commons Attribution Noncommercial License which permits any noncommercial use, distribution, and reproduction in any medium, provided the original author(s) and source are credited.

\section{References}

1. Budoff MJ, Dowe D, Jollis JG, Gitter M, Sutherland J, Halamert E, et al. Diagnostic performance of 64-multidetector row coronary computed tomographic angiography for evaluation of coronary artery stenosis in individuals without known coronary artery disease: results from the prospective multicenter ACCURACY (Assessment by Coronary Computed Tomographic Angiography of Individuals Undergoing Invasive Coronary Angiography) trial. J Am Coll Cardiol 2008;52:1724-32.

2. Meijboom WB, Meijs MF, Schuijf JD, Cramer MJ, Mollet NR, van Mieghem CA, et al. Diagnostic accuracy of 64-slice computed tomography coronary angiography: A prospective, multicenter, multivendor study. J Am Coll Cardiol 2008;52:2135-44.

3. Miller JM, Rochitte CE, Dewey M, Arbab-Zadeh A, Niinuma H, Gottlieb I, et al. Diagnostic performance of coronary angiography by 64-row CT. N Engl J Med 2008;359:2324-36.

4. Ostrom MP, Gopal A, Ahmadi N, Nasir K, Yang E, Kakadiaris I, et al. Mortality incidence and the severity of coronary atherosclerosis assessed by computed tomography angiography. J Am Coll Cardiol 2008;52:1335-43.

5. van Werkhoven JM, Schuijf JD, Gaemperli O, Jukema JW, Kroft $\mathrm{LJ}$, Boersma E, et al. Incremental prognostic value of multi-slice computed tomography coronary angiography over coronary artery calcium scoring in patients with suspected coronary artery disease. Eur Heart J 2009;30:2622-9.

6. Min JK, Shaw LJ, Devereux RB, Okin PM, Weinsaft JW, Russo DJ, et al. Prognostic value of multidetector coronary computed tomographic angiography for prediction of all-cause mortality. J Am Coll Cardiol 2007;50:1161-70.

7. Carrigan TP, Nair D, Schoenhagen P, Curtin RJ, Popovic ZB, Halliburton S, et al. Prognostic utility of 64-slice computed tomography in patients with suspected but no documented coronary artery disease. Eur Heart J 2009;30:362-71.

8. Kim TH, Hur J, Kim SJ, Kim HS, Choi BW, Choe KO, et al. Twophase reconstruction for the assessment of left ventricular volume and function using retrospective ECG-gated MDCT: Comparison with echocardiography. AJR Am J Roentgenol 2005;185:319-25.

9. Henneman MM, Schuijf JD, Jukema JW, Holman ER, Lamb HJ, de Roos A, et al. Assessment of global and regional left ventricular function and volumes with 64-slice MSCT: A comparison with 2D echocardiography. J Nucl Cardiol 2006;13:480-7.

10. Yamamuro M, Tadamura E, Kubo S, Toyoda H, Nishina T, Ohba $\mathrm{M}$, et al. Cardiac functional analysis with multi-detector row CT and segmental reconstruction algorithm: Comparison with echocardiography, SPECT, and MR imaging. Radiology 2005;234: 381-90.

11. de Feyter PJ, van Eenige MJ, Dighton DH, Visser FC, de JJ, Roos JP. Prognostic value of exercise testing, coronary angiography and left ventriculography 6-8 weeks after myocardial infarction. Circulation 1982;66:527-36.

12. Devereux RB, Roman MJ, Palmieri V, Liu JE, Lee ET, Best LG, et al. Prognostic implications of ejection fraction from linear echocardiographic dimensions: The strong heart study. Am Heart J 2003; $146: 527-34$

13. Hundley WG, Morgan TM, Neagle CM, Hamilton CA, Rerkpattanapipat $\mathrm{P}$, Link KM. Magnetic resonance imaging determination of cardiac prognosis. Circulation 2002;106:2328-33.

14. Sharir T, Germano G, Kavanagh PB, Lai S, Cohen I, Lewin HC, et al. Incremental prognostic value of post-stress left ventricular ejection fraction and volume by gated myocardial perfusion single photon emission computed tomography. Circulation 1999;100:1035-42.

15. van Werkhoven JM, Schuijf JD, Gaemperli O, Jukema JW, Boersma E, Wijns W, et al. Prognostic value of multislice computed tomography and gated single-photon emission computed tomography in patients with suspected coronary artery disease. J Am Coll Cardiol 2009;53:623-32.

16. Cademartiri F, Nieman K, van der Lugt A, Raaijmakers RH, Mollet N, Pattynama PM, et al. Intravenous contrast material 
administration at 16-detector row helical CT coronary angiography: Test bolus versus bolus-tracking technique. Radiology 2004:233:817-23.

17. Schuijf JD, Wijns W, Jukema JW, Atsma DE, de Roos A, Lamb $\mathrm{HJ}$, et al. Relationship between noninvasive coronary angiography with multi-slice computed tomography and myocardial perfusion imaging. J Am Coll Cardiol 2006;48:2508-14.

18. Leschka S, Husmann L, Desbiolles LM, Gaemperli O, Schepis T, Koepfli $\mathrm{P}$, et al. Optimal image reconstruction intervals for noninvasive coronary angiography with 64-slice CT. Eur Radiol 2006;16:1964-72.

19. de Graaf FR, Schuijf JD, van Velzen JE, Kroft LJ, de Roos A, Reiber JH et al. Diagnostic accuracy of 320-row multidetector computed tomography coronary angiography in the non-invasive evaluation of significant coronary artery disease. Eur Heart J 2009 (in press).

20. de Graaf FR, Schuijf JD, van Velzen JE, Nucifora G, Kroft LJ, de Roos A, et al. Assessment of global left ventricular function and volumes with 320-row multidetector computed tomography: A comparison with 2D-echocardiography. J Nucl Cardiol 2010;17: 225-31.

21. Thygesen K, Alpert JS, White HD. Universal definition of myocardial infarction. Eur Heart J 2007;28:2525-38.

22. Bassand JP, Hamm CW, Ardissino D, Boersma E, Budaj A, Fernandez-Aviles F, et al. Guidelines for the diagnosis and treatment of non-ST-segment elevation acute coronary syndromes. Eur Heart J 2007;28:1598-660

23. Lin FY, Devereux RB, Roman MJ, Meng J, Jow VM, Jacobs A, et al. Cardiac chamber volumes, function, and mass as determined by 64-multidetector row computed tomography: Mean values among healthy adults free of hypertension and obesity. JACC Cardiovasc Imaging 2008;1:782-6.
24. Hachamovitch R, Rozanski A, Hayes SW, Thomson LE, Germano G, Friedman JD, et al. Predicting therapeutic benefit from myocardial revascularization procedures: Are measurements of both resting left ventricular ejection fraction and stress-induced myocardial ischemia necessary? J Nucl Cardiol 2006;13:768-78.

25. Schulman SP, Achuff SC, Griffith LS, Humphries JO, Taylor GJ, Mellits ED, et al. Prognostic cardiac catheterization variables in survivors of acute myocardial infarction: A five year prospective study. J Am Coll Cardiol 1988;11:1164-72.

26. Min JK, Lin FY, Dunning AM, Delago A, Egan J, Shaw LJ, et al. Incremental prognostic significance of left ventricular dysfunction to coronary artery disease detection by 64-detector row coronary computed tomographic angiography for the prediction of all-cause mortality: Results from a two-centre study of 5330 patients. Eur Heart J 2010;31:1212-9.

27. Chow BJ, Wells GA, Chen L, Yam Y, Galiwango P, Abraham A, et al. Prognostic value of 64-slice cardiac computed tomography severity of coronary artery disease, coronary atherosclerosis, and left ventricular ejection fraction. J Am Coll Cardiol 2010;55: 1017-28.

28. Einstein AJ, Henzlova MJ, Rajagopalan S. Estimating risk of cancer associated with radiation exposure from 64-slice computed tomography coronary angiography. JAMA 2007;298:317-23.

29. Husmann L, Valenta I, Gaemperli O, Adda O, Treyer V, Wyss CA, et al. Feasibility of low-dose coronary CT angiography: First experience with prospective ECG-gating. Eur Heart J 2008;29: 191-7.

30. Shuman WP, Branch KR, May JM, Mitsumori LM, Lockhart DW, Dubinsky TJ, et al. Prospective versus retrospective ECG gating for 64-detector CT of the coronary arteries: Comparison of image quality and patient radiation dose. Radiology 2008;248:431-7. 